\title{
Research facilities and highlights at the Centro Nacional de Aceleradores (CNA)
}

\section{J. Gómez-Camacho ${ }^{1,2, a} \mathbb{D}$, J. García López ${ }^{1,2}$, C. Guerrero ${ }^{1,2}$,}

J. M. López Gutiérrez ${ }^{1,3}$, R. García-Tenorio ${ }^{1,4}$, F. J. Santos-Arévalo ${ }^{1}$, E. Chamizo ${ }^{1}$, F. J. Ferrer ${ }^{1}$, M. C. Jiménez-Ramos ${ }^{1}$, M. Balcerzyk ${ }^{1,5}$, B. Fernández ${ }^{1,2}$

${ }^{1}$ Centro Nacional de Aceleradores (U. Sevilla, J. Andalucía, CSIC), Avda Tomas A Edison, 7, 41092 Sevilla, Spain

2 Departamento de FAMN, U. Sevilla, Aptdo 1065, 41012 Sevilla, Spain

${ }^{3}$ Departamento de Física Aplicada I, Escuela Politécnica Superior, c/Virgen de África, 7, 41092 Sevilla, Spain

${ }^{4}$ Departamento de Física Aplicada II, ETS Arquitectura, Avda. Reina Mercedes, 2, 41012 Sevilla, Spain

5 Departamento de Fisiología Médica y Biofísica, Facultad de Medicina, Universidad de Sevilla, Avda. Sánchez Pizjuan, s/n, 41009 Sevilla, Spain

Received: 26 November 2020 / Accepted: 18 February 2021

(C) The Author(s) 2021

\begin{abstract}
The Centro Nacional de Aceleradores is a user-oriented accelerator facility in Seville, Spain. Its main facilities are a $3 \mathrm{MV}$ tandem accelerator, an $18 \mathrm{MeV}$ proton Cyclotron, a tandetron used for AMS, a compact accelerator used for radiocarbon measurements, a ${ }^{60} \mathrm{Co}$ irradiator and a PET/CT scanner. The technical specifications and research applications of these facilities are described. A neutron beam line associated to a charged pulsed beam in the tandem allows for time of flight measurements which determine the neutron energy. The use of an adequate stripper gas in the AMS tandetron permits to measure heavy radionuclides with very low detection levels, allowing to perform environmental studies using these radionuclides as tracers. The use of the microbeam in the tandem accelerator allows to apply the ion beam-induced current technique to investigate the spectroscopic properties and radiation hardness of different semiconductor detectors.
\end{abstract}

\section{Introduction}

The Centro Nacional de Aceleradores (CNA) is a joint research center of the University of Seville, the regional government of Andalucía and the Spanish National Research Council CSIC. It is recognized by the Spanish government as a Singular Scientific-Technical Facility, ICTS, dedicated to interdisciplinary research and open to external users. It has six major facilities: a Van de Graaff 3 MV Tandem accelerator, a 18/9 MeV Cyclotron accelerator, an Accelerator 1MV Mass Spectrometer, a PET / CT scanner, a radiocarbon dating system called $\mathrm{MiCaDaS}$ and a ${ }^{60} \mathrm{Co}$ Irradiator.

The application of these 6 infrastructures covers fields as diverse as material sciences, environmental impact, nuclear and particle physics, nuclear instrumentation, medical imag-

\footnotetext{
a e-mail: gomez@us.es (corresponding author)
} 
ing, biomedical research and preclinical molecular imaging, ${ }^{14} \mathrm{C}$ dating and irradiation in samples of technological and biological interest, among others.

CNA has a staff of about 57 from which 28 are PhD researchers, 18 technicians and 11 are PhD students. CNA has an external scientific committee, which advises on the scientific policy of the centre, and informs the proposals submitted by external users. The number of beam time applications carried our each year is about 100. The number of ISI papers per year is about 35 and the number of $\mathrm{PhD}$ thesis is 2-3. CNA funds come from the patron institutions, contracts with private companies and infrastructure and research projects. The local group of CNA, constituted by $28 \mathrm{PhD}$ researchers and their students, develops a vigorous research activity, both at CNA and in international centres, obtains competitive research funds coming from European, national and regional projects and successfully collaborates with the external users.

\section{Major facilities at CNA}

\subsection{Tandem accelerator of $3 \mathrm{MV}$}

The Tandem Accelerator, which was installed in 1998, was the first ion beam accelerator in Spain. It is a Pelletron 3 MV Tandem, model 9SDH-2, manufactured by National Electrostatics Corporation (NEC) which is capable of accelerating virtually all types of stable ions by applying a large potential difference of up to 3 million volts (MV). This machine is employed for characterization and modification of materials by means of ion beam analytical (IBA) techniques and ion implantation, as well as for the study and development of nuclear instrumentation, especially radiation detectors.

Negative ions are produced by three ion sources: (i) The Alphatross source is based on radiofrequency techniques and generates negative ions from gases $(\mathrm{H}, \mathrm{He}, \mathrm{N}, \ldots)$ in conjunction with an Rb charge-exchange channel. (ii) The SNICS II is a Cs sputtering source capable of producing tens of $\mu \mathrm{A}$ of negative ions from solid targets. (iii) The Duoplasmatron source, with a displaced intermediate (zwischen) electrode, provides modest currents of negative ions $\left(\mathrm{H}^{-}, \mathrm{C}^{-}, \mathrm{O}^{-}\right)$with a high-brightness, which is especially important for microbeam applications. The ion sources are connected to an injection magnet that selects the desired ion mass and directs the singly charged negative ions towards the entrance of the $3 \mathrm{MV}$ Tandem. Using a charge-changing process in the terminal, based on low pressure $\mathrm{N}_{2}$ gas, the ions are accelerated through the same high potential twice.

The beam transport system includes elements for focusing (magnetic quadrupoles and electrostatic lens), steering and monitoring (Beam Profile Monitors, Faraday Cups) the beam, a Wien filter and a 90 degrees analyzing magnet. At the end of the line, the beam can pass directly to the 0-degree line or can be deflected by a switch magnet with seven ports (Fig. 1).

At the present time, the 3 MV Tandem accelerator has eight available beamlines or experimental chambers.

1. Nuclear physics beam line This line includes a high-volume vacuum chamber, where nuclear instrumentation (detectors, electronics, etc), that will be used in international Nuclear Physics facilities, can be developed and tested [1].

2. Microbeam chamber Manufactured by Oxford Microbeam Ltd., in this line it is possible to focus the beam down to size of a few microns using a magnetic quadrupole triplet. The scanning system, synchronized with the data acquisition, allows the formation of maps from different signals with a maximum size of a few $\mathrm{mm}^{2}$. One of our main research lines 


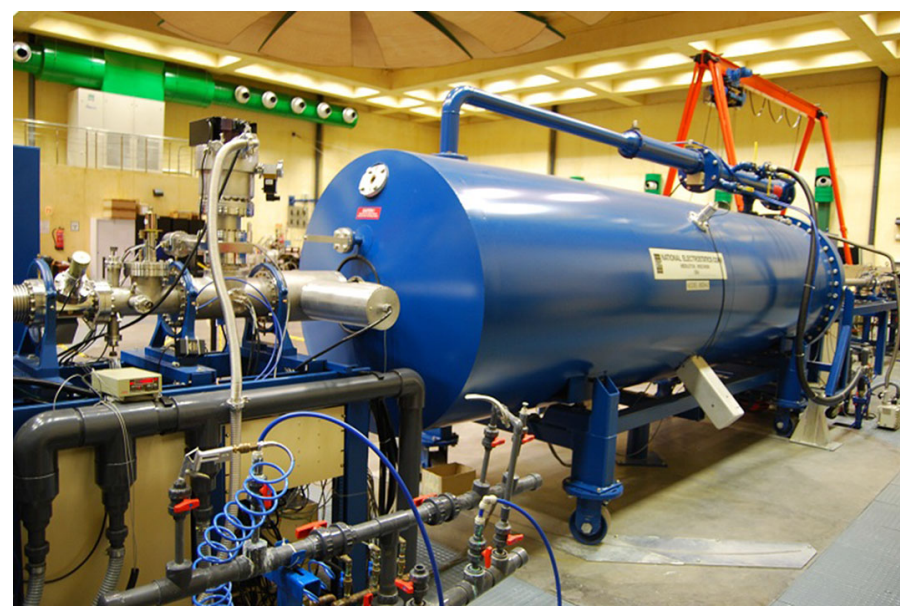

Fig. 1 Tandem accelerator

is the analysis by PIXE and RBS of the actinide elemental distribution in microscopic particles originating from the hydrogen bombs involved in several nuclear accidents [2], where the results are similar to those obtained by confocal X-Ray fluorescence (XRF) in a synchrotron facility.

3. Multipurpose IBA chamber This chamber is equipped with a set of particle, gamma and X-Ray detectors and a large target holder to carry out simultaneously different IBA experiments (RBS, PIXE, NRA and PIGE). In this chamber, we have recently investigated the use of novel solid ${ }^{4} \mathrm{He}$ targets for experimental studies on nuclear reactions $[3,4]$, the composition of solid-state hole conductor in solar cells prepared by vacuum processing [5] and a novel ionizing particle detector based on thin films multilayers [6,7].

4. Ionoluminescence chamber Located behind the multipurpose chamber, this vacuum chamber has black coating walls and is equipped with a heatable sample holder up to $500{ }^{\circ} \mathrm{C}$ and a photonic diagnostic system that allows mainly ionoluminescence studies. The first application of the chamber has been the characterization of different scintillator materials used for fast-ion loss detectors in nuclear fusion reactors [8].

5. Irradiation chamber This beamline has been designed to allow the irradiation of large areas (up to $16 \times 20 \mathrm{~cm}^{2}$ ) by raster scanning of the beam through magnetic deflection. It is mainly used by companies and research centers to perform irradiation tests of electronic devices and to test radiation detectors [9].

6. Channeling chamber Manufactured by Charles-Evans, this line is dedicated to channeling analysis of single crystalline samples using a 4-axis goniometer. A parallel beam is obtained with a telescopic system formed by two sets of slits. The chamber is equipped with particle, X-Ray and $\gamma$-Ray detectors. Through experiments in the implantation and channeling beamlines, we have recently studied the formation of magnetic SiC substrates for spintronic applications [10].

7. External beam This line is mainly used for Cultural heritage studies, since the use of in-air ion beam techniques (in combination with a good lateral resolution) presents numerous advantages for the analysis of inhomogeneous objects. The use of some elements purchased from Oxford Microbeams, like a magnetic quadrupole doublet, a precision fourjaw object slit and an exit nozzle set with micrometer adjustment, allow to obtain a spatial 
resolution of about $60 \mu \mathrm{m}$. Recent applications include the study of the manufacturing of gold jewels of the Carambolo treasure [11] and of gold electroplating techniques on silver substrates [12]

8. HiSPANoS line HiSPANoS, from Hispalis Neutron Source, is the first acceleratorbased neutron source in Spain. At HiSPANoS, neutrons are produced in a high-energy range covering from thermal to fast neutrons up to $9 \mathrm{MeV}$ through the reactions $p\left({ }^{7} \mathrm{Li}, n\right), d\left({ }^{7} \mathrm{Li}, n\right), d(\mathrm{D}, n), p\left({ }^{9} \mathrm{Be}, n\right)$ and $d\left({ }^{9} \mathrm{Be}, n\right)$. The main research application of the new neutron source is related to astrophysics, medical physics, detector characterization, electrical devices irradiation for aerospace purposes and neutron radiography, among others. In 2018, a buncher and chopper system and a new experimental line dedicated to neutron time of flight (TOF) measurements were installed. The pulsing system was designed to produce protons and deuterons pulsed beams with a pulse width of 1-2 $\mathrm{ns}$ at the target position at a frequency that can be varied from $32.5 \mathrm{kHz}$ to $2 \mathrm{MHz}$ [13].

\subsection{8/9 MeV Cyclotron accelerator}

Installed in 2004, the 18/9 Cyclotron, manufactured by Ion Beam Applications (IBA, Belgium) was the second particle accelerator at CNA (Fig. 2). The Cyclone 18/9 accelerates protons and deuterons to 18 and $9 \mathrm{MeV}$, respectively. Seven out of the eight targets are devoted to the production of positron emitters, while in the eighth port an external beamline has been installed to perform experiments in Nuclear Physics. The extracted maximum beam intensities in the internal target ports are $80 \mu \mathrm{A}$ for protons and $35 \mu \mathrm{A}$ for deuterons. Moreover, it is possible to use the Dual Beam Mode, which consists in the simultaneous bombardment with the same particle of two targets that are located in opposite positions.

Positron emitting isotopes ${ }^{18} \mathrm{~F},{ }^{11} \mathrm{C},{ }^{13} \mathrm{~N},{ }^{15} \mathrm{O}$ are produced in the cyclotron targets. CNA can produce several other isotopes in liquid or gaseous targets within limits set by Spanish regulatory authority (Consejo de Seguridad Nuclear). The produced isotopes are transported by tubing to automated synthesis modules of radiopharmacy. CNA has an agreement with Curium Pharma for the production of Positron Emission Tomography (PET) radiopharmaceuticals. Curium produces commercially $\left[{ }^{18} \mathrm{~F}\right]$ fluorodeoxyglucose $\left(\left[{ }^{18} \mathrm{~F}\right] \mathrm{FDG}\right)$ and $\left[{ }^{18} \mathrm{~F}\right]$ fluoromethylcholine for oncological studies, $\left[{ }^{18} \mathrm{~F}\right]$ fluorodopa for diagnosis of Parkinson's disease and $\left[{ }^{18} \mathrm{~F}\right]$ florbetaben for diagnosis of Alzheimer's disease. Except of the last radiopharmaceutical, CNA has access to these for preclinical studies. In research part of radiopharmacy $\left[{ }^{18} \mathrm{~F}\right] \mathrm{NaF}$ for bone scans, $\left[{ }^{11} \mathrm{C}\right]$ methionine $\left(\left[{ }^{11} \mathrm{C}\right] \mathrm{Met}\right),\left[{ }^{18} \mathrm{~F}\right]$ fluorothymidine $\left(\left[{ }^{18} \mathrm{~F}\right] \mathrm{FLT}\right)$ and $\left[{ }^{18} \mathrm{~F}\right]$ fluoromisonidazole $\left(\left[{ }^{18} \mathrm{~F}\right] \mathrm{FMISO}\right)$ used in oncology diagnosis may be produced for the use in clinical and preclinical studies. Their clinical use needs previous approval of Spanish regulatory authority for the use in each considered patient. Research compounds such as $\left[{ }^{18} \mathrm{~F}\right] \mathrm{NaBF} 4[14,15],\left[{ }^{18} \mathrm{~F}\right]$ Amylovis [16] and several others were produced in this radiopharmacy for preclinical use.

In the external beam line of the cyclotron, we can do research which requires the use of protons and deuterons with energies above $6 \mathrm{MeV}$, which is the maximum energy these particles can reach in our 3 MV Tandem. The particle beam passes through a thin window and goes to the air before impacting on the target. For material analysis, this presents some advantages over the use of a vacuum chamber, such as the low background X-Ray spectra obtained by PIXE technique, the higher X-ray production cross sections for K-lines of heavy elements and the greatest depth of analysis $[17,18]$. On the other hand, the setup of some irradiation experiments is simplified, the temperature reached at the target is lower than working in vacuum, and the size of the samples to be irradiated is not limited by the dimensions of the chamber. A very important detail is the internal coating, with a layer of graphite of few 


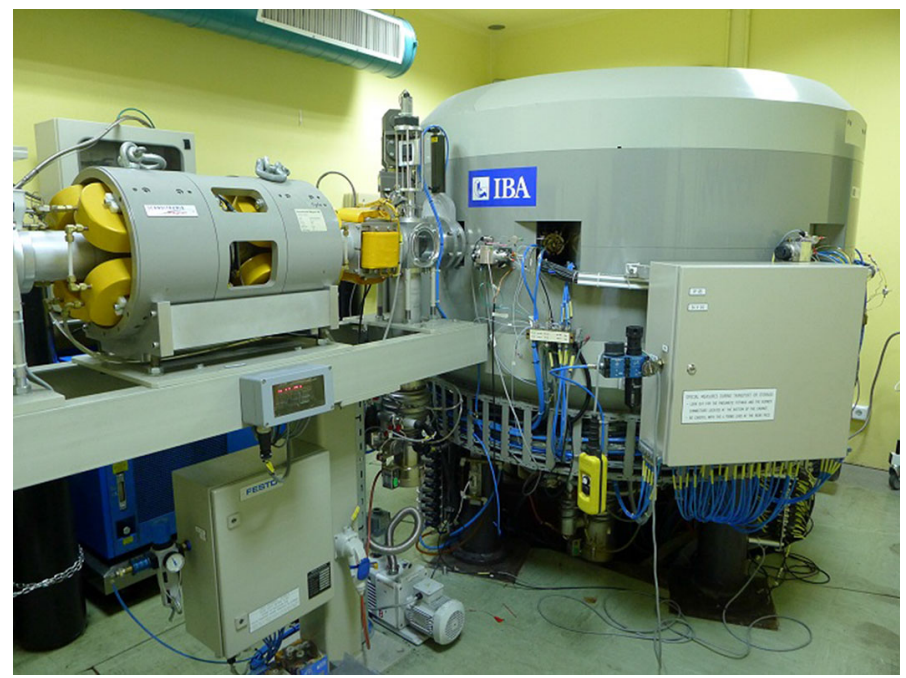

Fig. 2 Ciclotron accelerator

$\mathrm{mm}$ of thickness, of the metallic flange that forms the output window, to avoid its activation during the experiments. A motorized table with step motors, remotely controlled, allows the positioning of the targets with accuracy of $10 \mu \mathrm{m}$. Depending on the beam current intensity, this is monitored by different methods: current integrator, ionization chamber, silicon particle detector and radiochromic films. One of the advantages of the external line is its versatility; it can be modified with the necessary elements for each investigation without much complexity. For example, beam degraders, made of different materials and thicknesses, can be placed, both in vacuum and in air, to irradiate the samples with different energies and beam sizes.

\subsection{Accelerator 1MV Tandetron}

The Accelerator Mass Spectrometry (AMS) system was acquired in 2005 from the Dutch company High-Voltage Engineering Europe (HVEE). It is based on a 1 MV Tandetron accelerator (Fig. 3). AMS is an ultrasensitive technique dedicated to the detection of long-lived radionuclides which are present in very low amounts in the analyzed samples and that are beyond the scope of conventional radiometric and mass spectrometry techniques. Two properties give AMS this high sensitivity: First, molecules with the same mass as the isotope of interest are broken in the interaction with the stripper gas in the accelerator terminal. Second, using relatively high energies, the stopping power allows for additional isotope discrimination. Thanks to this, isotopic ratios as low as ${ }^{14} \mathrm{C} /{ }^{12} \mathrm{C}=10^{-16},{ }^{129} \mathrm{I} /{ }^{127} \mathrm{I}=10^{-14}$ or ${ }^{10} \mathrm{Be} /{ }^{9} \mathrm{Be}=10^{-14}$ can be achieved.

The 1MV AMS facility at CNA (SARA, from Spanish Accelerator for Radionuclide Analysis) consists firstly on a Cesium sputtering ion source for solid samples. Ions from the source are injected in the $1 \mathrm{MV}$ tandem through an injection magnet which separates the mass of interest. As described before, ions are stripped in the stripper channel, which is full of He gas at low pressure. This has shown to provide a much higher transmission for several isotopes than the original Ar specially in the case of the heaviest masses such as ${ }^{129} I$ and actinides. On the high energy side, the radioisotope of interest is conducted by an analyzing magnet and an electrostatic deflector to an ionization chamber. Stable isotopes are measured 


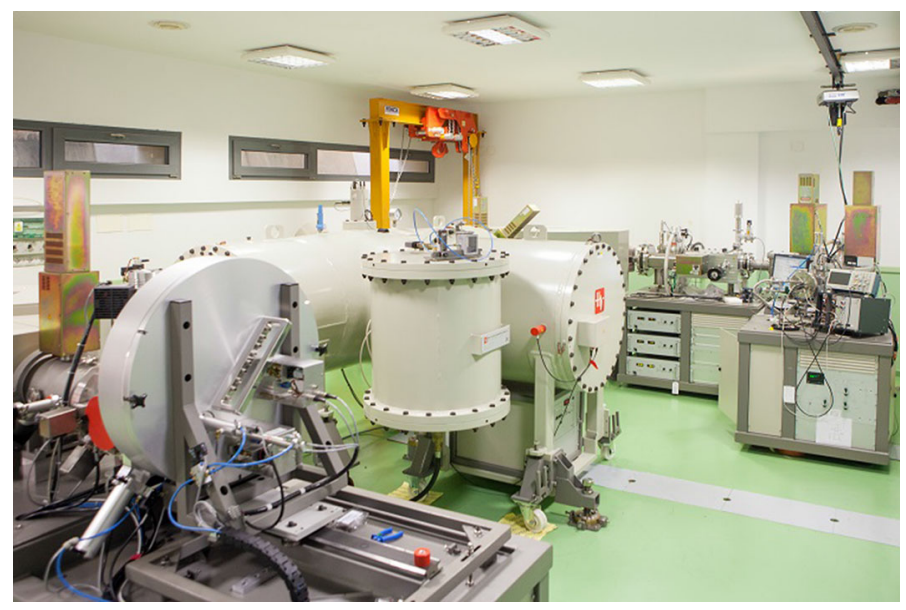

Fig. 3 AMS SARA accelerator

as an electric current after the magnet by a Faraday cup. Currently, ${ }^{10} \mathrm{Be},{ }^{14} \mathrm{C},{ }^{26} \mathrm{Al},{ }^{41} \mathrm{Ca},{ }^{129} \mathrm{I}$ and several actinides can be routinely detected at SARA $[19,20]$, which makes of this system a very versatile tool. For this reason, it can be applied to very different fields. At CNA, one of the most traditional and active lines is the study of environmental radioactivity. The ability to detect very small amounts of radionuclides in samples as water, air or soil allows to trace environmental processes. This can be applied not only to the distribution of radioactivity in the Biosphere but also to diverse problems as climate change, geology, hydrology, etc. For example, isotopes as ${ }^{129} \mathrm{I}$ or ${ }^{236} \mathrm{U}$ are being used at CNA to trace marine currents [21,22]. Also, sedimentation studies have been carried out through the measurement of ${ }^{10} \mathrm{Be}$ [23]. Apart from this, one of the latest applications of AMS at CNA is the characterization of nuclear residues. Thanks to its high sensitivity, AMS can measure very small concentrations of long-lived radionuclides in this kind of samples. This can help decide the final destination of the residues, which is critical for security and economical costs.

\subsection{MiCaDaS accelerator}

Since 2012, ${ }^{14}$ C Accelerator Mass Spectrometry measurements are performed in a dedicated system at CNA (Fig. 4). This system is called MiCaDaS (Mini Carbon Dating System) and was developed by the AMS group at the Ion Beam Physics Laboratory at the ETH (Federal Institute of Technology) in Zürich, Switzerland [24]. It is based on a $200 \mathrm{kV}$ tandem Accelerator. This terminal voltage is five times lower than the nominal terminal voltage at the analogue AMS system at CNA, SARA. It was designed for the exclusive detection of ${ }^{14} \mathrm{C}$, being currently commercialized by the Swiss company Ionplus.

As it was designed exclusively for ${ }^{14} \mathrm{C}$ measurement, several accessory focalization elements could be avoided, then reducing its size (only $2.6 \times 3.4 \mathrm{~m}^{2}$ ) and turning it into a simpler system. However, it follows the same structure of other AMS devices and is capable to obtain comparable results to other AMS facilities [25]. Graphite cathodes are prepared in the laboratory and ions are extracted in the ion source. After a first mass analysis, the beam is introduced in the accelerator. Afterwards, ions with charge state $q=1^{+}$are selected at the high energy side, where the final analysis of the beam components is performed. An 


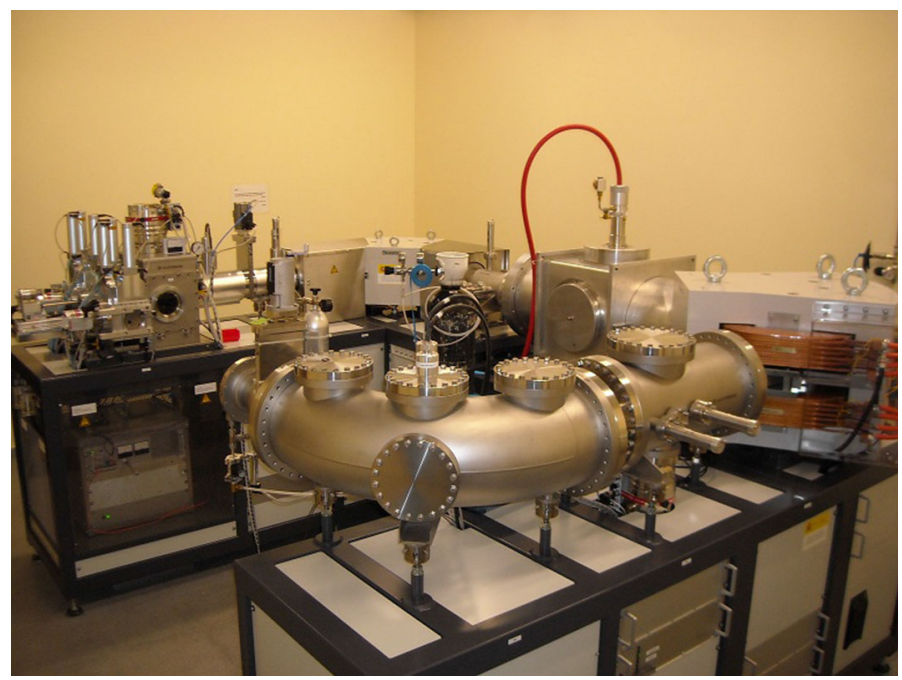

Fig. 4 MiCaDaS accelerator

ionization chamber gives information on the ${ }^{14} \mathrm{C}$ counts. As in other AMS systems, stable isotopes are quantified in the corresponding Faraday cups.

The MiCaDaS system includes a software package for sample preparation control. This software also controls the creation of sample batches for measurement and data analysis. It is optimized to work together with the AGE graphitization system, developed by the same group.

The very small sample amount needed permits Radiocarbon measurements performed with MiCaDaS in a variety of fields. For example, one single seed, or $1 \mathrm{~g}$ of bone are enough to get an accurate radiocarbon date. Then, it is easy to study archaelogical sites extensively, getting many dates from the same site, and thus obtaining much more valuable information [26]. The same can be applied to geological or environmental studies. As an example, it is possible to obtain information about deposition rates in sediments and peats, or information about landscape evolution [27] [28]. Also forensic applications have been carried out by the measurement of ${ }^{14} \mathrm{C}$, such as the dating of ivory samples against poaching and ivory smuggling. The nuclear bomb testing in the mid-twentieth century, labeled all the organic materials with very high ${ }^{14} \mathrm{C}$ concentrations. At CNA, this has been used to determine whether the ivory used in a sculpture is old enough for the sculpture to be legally traded [29].

\subsection{PET/CT clinical and preclinical scanners}

Complementary to the particle accelerators described above, CNA has clinical and preclinical scanners (Fig. 5). These instruments widen the research and applications scope of CNA and benefit from multiple synergies, associated to common aspects in radioprotection, detector technologies, production of novel PET isotopes the accelerators, etc. Research in this field is carried out by a small group of CNA personnel, which collaborates with external users and clinical personnel.

Since late 2011, the National Accelerator Centre has a Clinical PET/CT, which allows receiving patients at CNA facilities, particularly in the Diagnostic Imaging Center of CNA (CDI). Human scanner shares its control room with that of a small animal PET and CT scanner. 

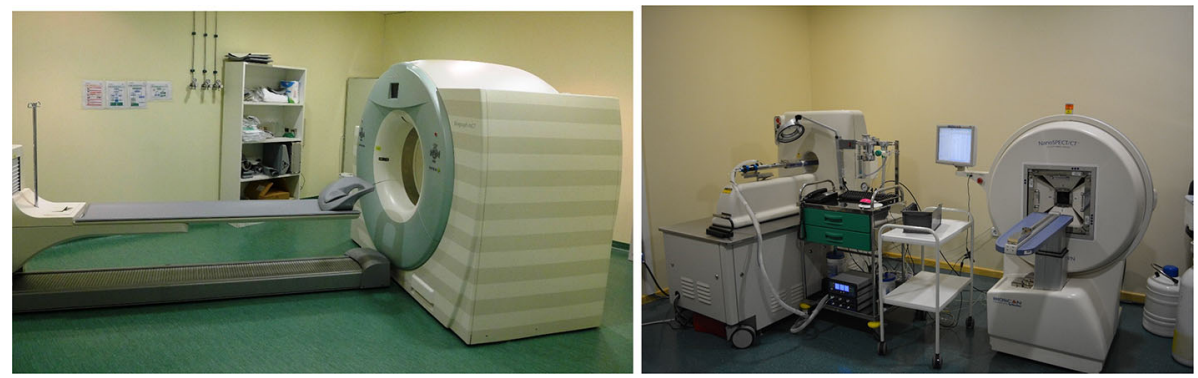

Fig. 5 Clinical PET-CT scanner (left) and preclinical micro-PET and nanoCT (right)

It is installed a few meters away from the radiopharmacy laboratory, which allows studies with short half-life PET radioisotopes, such as ${ }^{11} \mathrm{C}$-based radiopharmaceuticals. There is an agreement with the Nuclear Medicine Services of the University Hospitals Virgen del Rocio and Virgen Macarena to use and support the research facility in CNA. Presently, Hospital patients of research interest are scanned at CNA three days per week, and for the remaining two days the Service provides the qualified staff (Nuclear Medicine specialists, nursery and technicians staff) for the human studies proposed by non-Hospital scientific personnel. Moreover, the CT scanner is used for examination of art large objects and animals which due to their size or mass do not fit in the animal scanner.

Since 2005 CNA has a preclinical PET and CT scanners. Both PET and CT scanners can operate in multibed mode allowing for large animal scans. Mosaic PET images and NanoCT images can be co-registered in external PMOD software because the animal is immobilized in the heated bed and can be transferred between these two scanners without moving it in the bed. Dynamic and listmode PET images can be acquired in Mosaic PET. Clinical and preclinical PET were used in numerous studies with animal models of acromegaly [30] with $\left[{ }^{18} \mathrm{~F}\right] \mathrm{FDG}$ and $\left[{ }^{11} \mathrm{C}\right] \mathrm{Met}$, and of subarachnoid haemorrhage with $\left[{ }^{18} \mathrm{~F}\right] \mathrm{FMISO}$ [31], in collaboration with IBIS (Spain). With CENTIS (Cuba) we examined $\left[{ }^{18} \mathrm{~F}\right]$ Amylovis, a potential radiotracer for detection of $\beta$-Amyloid in Alzheimer's disease [32]. Studies contributing to the standardization of clinical PET/CT with [ ${ }^{18}$ F]FDG [33] and of radiotherapy planning [34] have been done. NanoCT was used for nanoparticle characterization in collaboration with ICMSE (Spain) [35], and also for cryopreservation studies in collaboration with UCL (Belgium) [36].

\subsection{Co-60 irradiator}

The proton and heavy ions irradiation research lines associated to the $3 \mathrm{MV}$ Tandem and 18 MV Cyclotron accelerators were complemented in 2013 with the incorporation to CNA of a system to perform irradiation studies with photons. This system, provided by Best Theratronics limited (model Gammabeam X200), is equipped with a ${ }^{60}$ Co source of 357 $\mathrm{TBq}$ (December 2013), which generates in air and at $100 \mathrm{~cm}$ distance a maximum kerma rate of $103 \mathrm{~Gy} / \mathrm{h}$.

With this new facility, the CNA in a first instance offers the possibility to perform reliability studies of electronic components used in the aerospace industry, but due to the versatility of the acquired system, it was possibly quickly to observe also the applicability of this irradiator to Materials Science in studies devoted to analyze thermal, electric or mechanical properties changes in materials allocated in radiation environments. 
The most recent applications of the $\mathrm{CNA}{ }^{60} \mathrm{Co}$ irradiator have been mainly focussed in to analyze dose effects in different electronic systems: unclonable functions implemented in FPGAs [37] and SIMD microprocessors [38,39].

The CNA ${ }^{60} \mathrm{Co}$ irradiation facility is in accordance with the European Space Agency Basic Specification ESCC 22900 (Total Dose Steady-State Irradiation Test Method), as well as with the Defense Logistics Agency tests methods MIL-STD-750 1019 and MIL-STD-883 1019 .

\section{Research highlights}

\subsection{Pulsed neutron beams at HiSPANoS}

The HiSPANoS neutron source features a number of neutron production mechanisms, as summarized in Table 1. Using continuous beams, before the 2018 upgrade, the main research applications of the neutron source were related to astrophysics [40,41], detectors characterization [42] and electrical devices irradiation for aerospace purposes [43], among others. Recently, a new research line in neutron radiography has been launched using both thermal and fast neutrons, producing images that combined with $\gamma$-radiographies using the ${ }^{60} \mathrm{Co}$ irradiator described above provide CNA with a unique set of imaging capabilities.

The mentioned 2018 upgrade allowed producing pulsed ion beams that provide time-offlight capabilities to HiSPANoS. The pulsing system from NEC consists of a chopper $(650 \mathrm{~V})$ that deflects the continuous beam after at the exit of the $60 \mathrm{kV}$ injector allowing the passage of pulses with a temporal width that can be varied between 40 and 250 ns. The repetition rate can also be adjusted, in this case between $32.2 \mathrm{kHz}$ to $2 \mathrm{MHz}$ and is usually chosen as high as possible but considering that there is no overlap between consecutive neutron pulses. For instance, a $1 \mathrm{keV}$ neutron needs $\sim 3.4 \mu$ s to travel the $150 \mathrm{~cm}$ that separate the neutron production target from a detector or sample placed at $150 \mathrm{~cm}$ distance, and hence time the repetition rate for epithermal neutrons is limited to about $250 \mathrm{kHz}$. Downstream from the chopper, the buncher module enables the compression of the beam pulses in such a way that the $40 \mathrm{~ns}$ pulse is reduced to only $1.7 \mathrm{~ns}$ (FWHM) at the end of the beam line, where the neutron production target of choice is placed. With this pulse width, the energy resolution achieved at a $150 \mathrm{~cm}$ flight path is $0.2 \%$ at $20 \mathrm{keV}, 1.5 \%$ at $1 \mathrm{MeV}$ and $5 \%$ at $12 \mathrm{MeV}$.

The neutron beams available for time-of-flight experiments are illustrated in Fig. 6. The epithermal neutron beam [13] is produced by the ${ }^{7} \mathrm{Li}(\mathrm{p}, \mathrm{n})$ reaction . This energy distribution, of interest in s- and r-process nucleosynthesis calculations, has been characterized in terms of intensity and angular distribution by means of a Li-glass detector at 35 and $50 \mathrm{~cm}$ flight path

Table 1 Targets and neutron production reactions available at HISPANOS

\begin{tabular}{lllcl}
\hline Target & Target description & Reaction & Q-value $(\mathrm{MeV})$ & Neutron energy \\
\hline $\mathrm{Li} / \mathrm{LiF}$ & Thick, water-cooled $/ 16 \mu \mathrm{m}$ on Cu backing & $\mathrm{p}\left({ }^{7} \mathrm{Li}, \mathrm{n}\right){ }^{7} \mathrm{Be}$ & -1.64 & epithermal \\
$\mathrm{Li} / \mathrm{LiF}$ & Thick, water-cooled $/ 16 \mu \mathrm{m}$ on Cu backing & $\mathrm{d}\left({ }^{7} \mathrm{Li}, \mathrm{n}\right)^{7} \mathrm{Be}$ & 15.03 & Up to $15 \mathrm{MeV}$ \\
$\mathrm{D}$ & $\mathrm{D} / \mathrm{Ti}$ ratio $\geq 1.5,500 \mu \mathrm{g} / \mathrm{cm}^{2}$ on Al backing & $\mathrm{d}(\mathrm{D}, \mathrm{n})^{3} \mathrm{He}$ & 3.27 & $\begin{array}{c}2.6-3.5 \mathrm{MeV} \\
\text { (monoenergetic) }\end{array}$ \\
$\mathrm{Be}$ & $2 \mathrm{~mm}$ thick & $\mathrm{d}\left({ }^{9} \mathrm{Be}, \mathrm{n}\right)^{10} \mathrm{~B}$ & 4.36 & Up to $8 \mathrm{MeV}$ \\
\hline
\end{tabular}

The corresponding neutron fluxes can be calculated with the tools NeuSDesc and PINO 

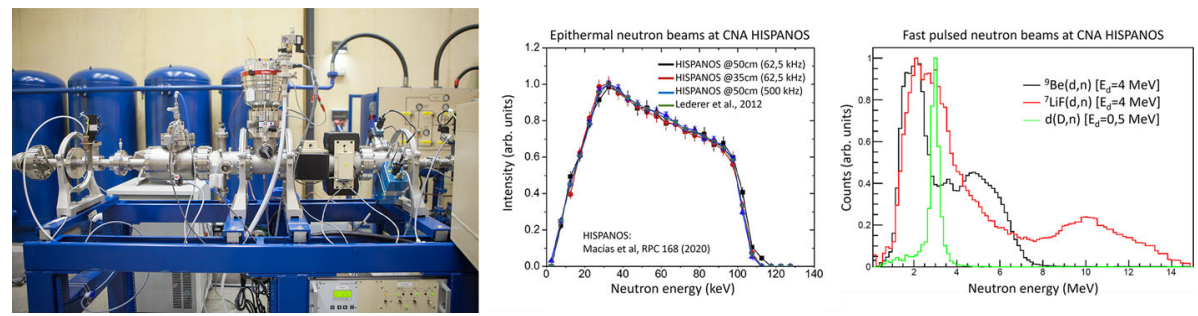

Fig. 6 Neutron beam line, neutron energy distribution of the epithermal (from Ref. [13]) and fast pulsed neutron beams produced at HiSPANoS. See text for more details

distances. The results [44] agree with the evaluation of ref. [45]. The fast neutron beams are produced from deuteron beams incident on the different targets available. The figure shows results from a measurement at a flight path of $150 \mathrm{~cm}$ with a EJ301 liquid scintillator featuring pulse shape discrimination capabilities. Noticeable features include: (i) the maximum neutron energy achievable at HiSPANoS is $\sim 15 \mathrm{MeV}$, reached by means $5 \mathrm{MeV}$ deuterons hitting the $\mathrm{LiF}$ target, (ii) monoenergetic neutron beams can be produced via the $\mathrm{d}(\mathrm{D}, \mathrm{n})$ fusion reaction, and (iii) the maximum neutron flux is achieved via $\mathrm{d}\left({ }^{9} \mathrm{Be}, \mathrm{n}\right)$ reactions on a thick Be target. The walls of the facility are thick enough ( $1 \mathrm{~m}$ concrete) to run experiments with neutrons at the maximum achievable production rates without posing radiological hazards outside the experimental hall.

At HiSPANoS the external users have at their disposal a set of detectors and a digital (8 channels, $2 \mathrm{GS} / \mathrm{s}$ and 10 bits) data acquisitions system. Detectors available include a neutron dosimeter, plastic and liquid (with Pulse Shape Discrimination capabilities) scintillators for fast neutrons, Li-glass detectors of different thicknesses for thermal and epithermal neutrons, and $\mathrm{LaBr}_{3}$ and HPGe detectors for $\gamma$-rays. Considering that the closest wall is at $5 \mathrm{~m}$ from the neutron production target, the flight paths available are up to $2 \mathrm{~m}$ in the forward direction and up to $4 \mathrm{~m}$ at 60 degrees. The access to the facility for international users is funded until 2024 via the H2020 ARIEL project's Transnational Access program.

\subsection{Actinides measurements on the 1 MV AMS system}

The Pu AMS measurement technique (i.e., ${ }^{239} \mathrm{Pu}$ and ${ }^{240} \mathrm{Pu}$ ) was successfully implemented at CNA 12 years ago. It was the second time that a compact AMS facility, operating with acceleration terminal voltages below $1 \mathrm{MV}$ and featuring a compact design, proved its potential to perform such demanding measurements. Subsequently, the system was set up for ${ }^{236} \mathrm{U}$ measurements with very competitive ${ }^{236} U /{ }^{238} U$ background levels $\left(3 \cdot 10^{-11}\right)$. However, ${ }^{236} \mathrm{U} /{ }^{238} \mathrm{U}$ atom ratio levels at the $10^{-13}$, which are the minimum ones that can be found in natural samples, were not attainable. They could only be achieved by optimized AMS setups with additional kinematic filters. The implementation of He as stripper gas in 2015 meant a significant improvement in the measurement conditions for actinides. Stripping yields for $3^{+}$charge state at low energies, progressed from $13 \%$ for former Ar gas to $50 \%$ for He, thus increasing the counting yields, improving the quality of the AMS results and opening the gate to minor actinides isotopes such as ${ }^{241} \mathrm{Pu}$ (Fig. 7). However, ${ }^{236} \mathrm{U}$ with He presented worse ${ }^{236} U /{ }^{238} U$ background ratios than with Ar, at the $10^{-10}$ level, due to the enhancement of the scattering processes with residual He gas, more mobile than Ar, occurring along the high-energy acceleration tube and causing further background events from ${ }^{235} \mathrm{U}$ and ${ }^{238} \mathrm{U}$ molecular fragments. The last actinide to be incorporated to the CNA catalog is ${ }^{237} \mathrm{~Np}$, with a 


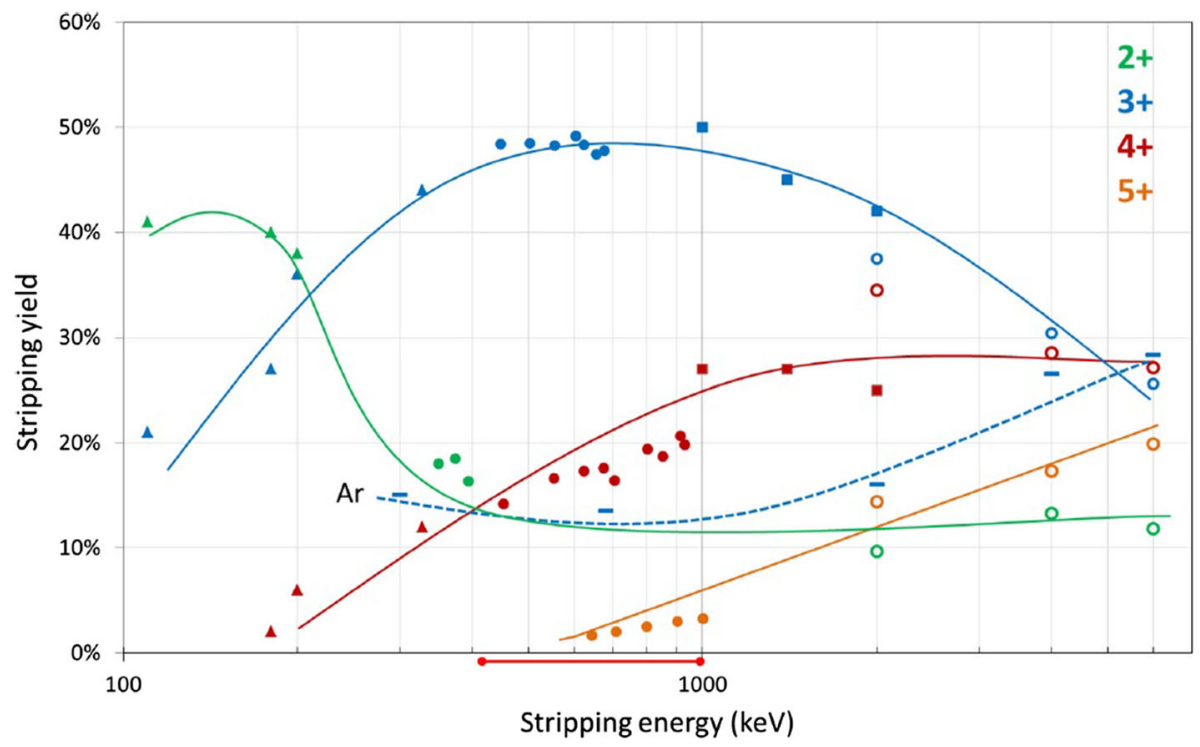

Fig. 7 U stripping reported by different AMS facilities in different energy ranges using He gas as stripper. CNA results, which correspond to the circles (403-1000 keV range), can be compared with the full line, which is the interpolated results from interpolated other institutes (complete reference in [46])

Table 2 AMS isotops available at CNA-SARA

\begin{tabular}{lllll}
\hline Stripper gas & Radionuclide & Transmission (\%) & Background (ratio to 238U) & Instrumental blank (atoms) \\
\hline $\mathrm{He}$ & $237 \mathrm{~Np}$ & 40 & $5.10^{-11}$ & $<10^{6}$ \\
& $239 \mathrm{Pu}$ & & $3.10^{-9}$ & \\
& $240 \mathrm{Pu}$ & & $7.10^{-10}$ & \\
& $236 \mathrm{U}$ & 30 & $8-13 \cdot 10^{-11}$ & \\
$\mathrm{Ar}$ & $239 \mathrm{Pu}$ & 13 & $4.10^{-9}$ & \\
& $240 \mathrm{Pu}$ & & $3.10^{-11}$ & \\
& $236 \mathrm{U}$ & & $3.10^{-11}$ & \\
\hline
\end{tabular}

very competitive limit of detection (at the $10^{6}$ atoms level) achieved in natural samples with significant amounts of ${ }^{238} \mathrm{U}$. The key point here has been the combination of a thoroughly optimized tuning of the system (i.e., slits settings and stripper gas pressure) with a careful sample processing aimed at minimizing $U$ background $[46,47]$. A summary of the actinides available at CNA is presented in Table 2, indicating the transmission through the accelerator, as well as the background, and the instrumentation blank level, which is below $10^{6}$ atoms per sample in all cases.

To date, CNA has consolidated its position as a competitive facility in the actinides measurement community. The implementation of state-of-the-art radio-chemical methods has allowed us to afford the measurement of ${ }^{239,240,241} \mathrm{Pu},{ }^{236} \mathrm{U}$ and ${ }^{237} \mathrm{~Np}$ in a wide variety of environmental samples, and new results have come to light. Most recent studies have been performed in the frame of the existing collaboration with the IAEA environmental laboratories. Worth of mention are the studies carried out in the Namibian coast, where ${ }^{236} \mathrm{U},{ }^{237} \mathrm{~Np}$ 
and ${ }^{239,240} \mathrm{Pu}$ levels and sources have been assessed for the first time in the African coast, pointing out to global fallout as their main source (i.e., baseline levels from the large-scale testing of nuclear weapons in the 1945-1980 period) [22]. On the other hand, though, for the first time, the study of the ${ }^{237} \mathrm{~Np} /{ }^{236} \mathrm{U}$ signal, the sources of actinides to the Mediterranean Sea and to the Canada Basin in the Arctic Ocean have been approached [48]. Another recent study points out to the tropospheric fallout from the British and French nuclear tests in the Southern Hemisphere as the main source of ${ }^{239,240,241} \mathrm{Pu}$ to Madagascar [49]. New techniques will be tested in a future to improve the CNA actinides measurement conditions.

\subsection{IBIC technique in the microprobe}

Since 2012, the ion beam-induced current (IBIC) technique is available at CNA, which has led to a series of new collaborations with national and international groups working in the field of radiation detectors. In an IBIC experiment, a rarefied $\mathrm{MeV}$ light ion beam (a few hundred or thousand ions per second) is used to analyze the active regions of a semiconductor device. The charge induced by the motion of carriers generated by each ion is detected, amplified and processed using a conventional electronic chain. Another possibility is to record the signal produced in the detector directly on a fast oscilloscope, so that instead of measuring only the total induced charge, the time profile of the current pulse is also obtained. In that case the technique is called Time-Resolved IBIC or TRIBIC [50,51]: In both modalities, it is generally advantageous to perform the experiments on an ion microprobe line, not only to make use of a good spatial resolution of the order of the micron, but to be able to control and drastically reduce the ion rate used during the measurements in order to avoid, as far as possible, introducing ion-induced damage.

In the frame of a Coordinated Research Project from the International Atomic Energy Agency (IAEA) entitled "Utilization of ion accelerators for studying and modeling of radiation induced defects in semiconductors and insulators," we have collaborated in the development of a standardized methodology to quantify the radiation hardness of semiconductor devices $[52,53]$. The procedure includes the irradiation with $\mathrm{MeV}$ light ions at different fluences of the device under study (DUS) and subsequent IBIC measurement to determine the degradation of the Charge Collection Efficiency (CCE) due to the induced structural damage. Then, the analysis of the experimental results is carried out using a theoretical approach based on the ionization and non-ionization energy loss in solids, the theory of charge induction in semiconductors and the Shockley-Read-Hall statistics for the carrier recombination in the presence of deep traps. As an example of this methodology, we have compared the decrease in the carrier lifetimes of $\mathrm{Si}$ and $\mathrm{SiC}$ diodes after irradiation with $17 \mathrm{MeV}$ protons. Unexpectedly, it was observed that the degradation is considerably larger in the case of $\mathrm{SiC}$ (considered as a radiation hard material) but this effect is counteracted by the much larger electric field present in the $\mathrm{SiC}$ samples and the shorter distance that the carriers have to cross in these detectors [54].

IBIC characterization of single-crystal diamond detectors developed for neutron spectroscopy applications was done in collaboration with the Rutherford Appleton Laboratory (UK) and the Università degli Studi di Milano-Bicocca (Italy). Detectors with large contacts present good charge collection uniformity, while edge effects are important for detectors equipped with a small contact [55]. CNA micro-beam was also used to study irradiation effects that can hinder the normal operation of a diamond spectrometer, as polarization effects, which could be recovered with polarity cycles and irradiation. IBIC and TRIBIC techniques were applied to advanced silicon detectors developed at Instituto de Microelectrónica de Barcelona (IMB-CNM, CSIC). Using microbeams of $\mathrm{He}^{2+}$ and $\mathrm{H}^{+}$, charge collection studies of a new 


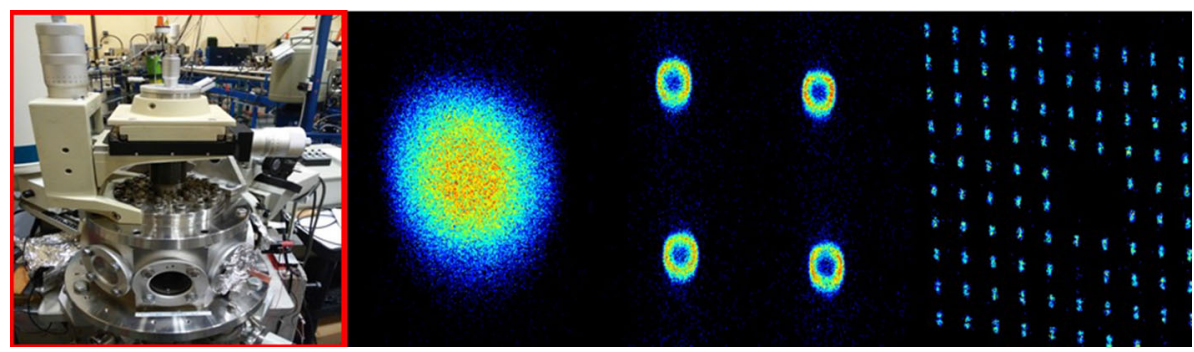

Fig. 8 Left: Nuclear microbeam chamber. Right: IBIC maps with $5 \mathrm{MeV} \mathrm{He}^{2+}$ in microdosimeters [56]

generation of cylindrical 3D silicon structures were performed [56]. IBIC maps showed a $100 \%$ yield of active cells in a microdosimeter array, with each microsensor acting as an independent active site (Fig. 8). Also, IBIC measurements proved to be a powerful method to characterize the active area and effective radius of these micrometer-sized sensors.

Since 2017 the CNA is a member of the international CERN RD-50 collaboration "Radiation hard semiconductor devices for very high luminosity colliders" that started in 2001 to develop radiation hard silicon detectors for the High luminosity LHC and other future colliders. CNA contributes to this collaboration with the first characterization by IBIC and TRIBIC of silicon low gain avalanche detectors (LGAD), developed by the IMB and the Instituto de Física de Cantabria (IFCA). The LGAD detectors present intrinsic gain due to the multiplication layer implanted below the cathode in which the electric field is enhanced significantly, this gain can reach a factor of 20 in pristine detectors. It is interesting to note that the gain vs. applied voltage curve obtained by IBIC is clearly lower than that measured using a laser, without the mechanism behind this discrepancy having been elucidated so far. The measurements we have made using ions with different stopping powers seem to indicate that this effect is probably not due to a partial shielding of the electric field because of the high density of carriers formed along the ion track.

Finally, the implementation of the IBIC technique at CNA is relevant for the business sector. SiC detectors with applications in nuclear fusion are being developed together with the company ALTER Technology and the IMB [57]. The advantage of this wide bandgap material over silicon is its capacity to work at high temperatures, which makes it a potential candidate for the detection of escaping 3.5 MeV He ions in future D-T-based fusion reactors. So far we have studied spectrometric and transport properties in SiC detectors at room temperature [57], and we are currently setting-up our facilities to be able to carry out IBIC studies at high temperature.

\section{Summary and outlook}

CNA is a users-oriented research centre where accelerators are used for a variety of crossdisciplinary applications. Here we have presented succinctly the six major facilities and discussed more in depth three recent research highlights.

The vision for the near future of CNA is established in the strategic plan for the centre. We want to expand our capabilities as a user-oriented facility, maintaining our status as a Spanish ICTS (singular scientific and technological facility), and promoting the access of national and international users. This requires an continuous upgrading of the facilities, but, even more importantly, to maintain the motivation of CNA staff to engage external users. We 
consider CNA as a unique facility in Spain to improve the training of University students, at the degree, master and doctorate levels.

We envisage CNA as an environment to promote the interaction between academics and high technology companies: CNA can provide services to companies, such as the irradiation with the ${ }^{60} \mathrm{Co}$ irradiator, or the production of PET isotopes in the cyclotron, while companies can provide equipment required to CNA, such as beam diagnostics and detector systems for the Tandem beam lines and the Cyclotron external line. CNA can provide hands-on training for companies' staff, while companies can provide professional opportunities for CNA young researchers.

We view CNA, as well as other small and medium sized facilities across Europe, as key actors in the European scientific and technological ecosystem. CNA maintains links with large, transnational facilities in Europe, which are more dedicated to fundamental science, specially in nuclear and particle physics. CNA is a place where researchers can be trained, equipment can be developed and tested, and scientific questions complementary to those addressed in large facilities, can be addressed. In this line, IBIC analytical capabilities, as well as HiSPANoS pulsed neutron beams, are indeed complementary to large facilities at CERN such as LHC and nToF.

Applications will indeed continue to be at the core of CNA future. Environmental studies, using the excellent AMS actinide measurements, as well as Cultural heritage studies, using the Tandem ion beam analysis and $\mathrm{MiCaDaS}$ radiocarbon dating, should expand in the future. Clinical and preclinical PET and CT imaging studies, done in collaboration with the medical staff coming to CNA, are carried out and should improve. Proton therapy-related studies, making use of CNA accelerators to irradiate cell cultures, have been done, and set a solid basis for a future CNA clinical proton therapy facility.

\begin{abstract}
Acknowledgements We would like to thank the effort of all the scientists, technicians and administrative personnel, as well as the external users, which have contributed to CNA since its beginning in 1997. Also the funding made available for infrastructures through the ICTS program of the Spanish ministry of science, making use of FEDER funds from the European Union. The continuous support of CNA patrons, the University of Seville, the Junta de Andalucía and CSIC is acknowledged. Private funds, through contracts with companies and institutions, such as Curium, Alter technologies, and the andalusian health service (SAS), made possible to maintain contracts of key personnel. We acknowledge the funding of projects by the European Union, H2020-847594, H2020-654002, H2020-847552, H2020-847594 , by the Spanish ministry of science RTI2018-098117-B-C21, RTC-2017-6369-3, EQC2018-004193-P, EQC2018-004095-P, EQC2018-004166P, PGC2018-094546-B-I00, and by the Junta de Andalucía FEDER progrem US-1261006, US-1263369, P18-RT-1900.
\end{abstract}

Open Access This article is licensed under a Creative Commons Attribution 4.0 International License, which permits use, sharing, adaptation, distribution and reproduction in any medium or format, as long as you give appropriate credit to the original author(s) and the source, provide a link to the Creative Commons licence, and indicate if changes were made. The images or other third party material in this article are included in the article's Creative Commons licence, unless indicated otherwise in a credit line to the material. If material is not included in the article's Creative Commons licence and your intended use is not permitted by statutory regulation or exceeds the permitted use, you will need to obtain permission directly from the copyright holder. To view a copy of this licence, visit http://creativecommons.org/licenses/by/4.0/.

\title{
References
}

1. A. Garzón-Camacho, B. Fernández, M.A.G. Alvarez, J. Ceballos, J.M. de la Rosa, IEEE Trans. Inst. Meas. 64, 318-327 (2015)

2. M.C. Jimenez-Ramos, M. Eriksson, J. García López, Y. Ranebo, R. García-Tenorio, M. Betti, E. Holm, Spectrochim. Acta Part B 65, 823-829 (2010) 
3. F.J. Ferrer, B. Fernández, J.P. Fernández-García, F.G. Barba, A. Fernández, D. Galaviz, V. Godinho, J. Gómez-Camacho, A.M. Sánchez-Benítez, Eur. Phys. J. Plus 135, 465 (2020)

4. V. Godinho, J. Caballero, A. Fernández, F.J. Ferrer, J. Gómez-Camacho, B. Fernández, Spanish patent No. ES2582052 (2018)

5. A. Barranco, M.C. Lopez-Santos, J. Idigoras, F.J. Aparicio, J. Obrero-Perez, V. Lopez-Flores, L. Contreras-Bernal, V. Rico, J. Ferrer, J.P. Espinos, A. Borras, J.A. Anta, J.R. Sanchez-Valencia, Adv. Energy Mater. 10, 1901524 (2019)

6. J. Gil-Rostra, F.J. Ferrer, J.P. Espinos, A.R. Gonzalez-Elipe, F. Yubero, ACS Appl. Mater. Interfaces 9 , 16313-16320 (2017)

7. J. Gil-Rostra, A.R. Gonzalez-Elipe, J.P. Espinos, F. Yubero, A. Barranco, F.J. Ferrer, J. Cotrino, Spanish patent No. ES2548912 (2016)

8. M.C. Jiménez-Ramos, J. García López, M. García-Muñoz, M. Rodríguez-Ramos, M. Carmona Gázquez, B. Zurro, Nucl. Instr. Meth. B 332, 216-219 (2014)

9. M. Seimetz et al., IEEE Trans. Nucl. Sci. 62(6), 3216-3224 (2015)

10. J. Garcia Lopez, Y. Morilla, J.C. Cheang Wong, G. Battistig, Z. Zolnai, J.L. Cantin, Nucl. Instr. Meth. B. 267, 1097-1100 (2009)

11. S. Scrivano et al., Radiat. Phys. Chem. 130, 133-141 (2017)

12. I. Ortega Feliu et al., Nucl. Instrum. Methods Phys. Res. Sect. B 406, 318-323 (2017)

13. M. Macias, B. Fernandez, J. Praena, Radiat. Phys. Chem. 168, 108538 (2020)

14. M. Balcerzyk, M. De-Miguel, C. Guerrero, B. Fernandez, Cells 9, 9 (2020)

15. M. Balcerzyk, L. Fernandez-Maza, J.J. Mínguez, M. De-Miguel, Jpn. J. Clin. Oncol. 48(2), 200 (2018)

16. S. Rivera-Marrero et al., Curr. Radiopharm. 12(1), 58 (2019)

17. J. Garcia Lopez, I. Ortega-Feliu, Y. Morilla, A. Ferrero, Nucl. Instr. Meth. B. 266, 1583-1586 (2008)

18. M.C. Jiménez-Ramos, J. García López, M. Eriksson, J. Jernstrom, R. García-Tenorio, Nucl. Instr. Meth. B. 273, 118-121 (2012)

19. E. Chamizo et al., Nucl. Instrum. Methods Phys. Res. Sect. B Beam Interact. Mater. Atoms 361, 13-19 (2015)

20. G. Scognamiglio et al., Nucl. Instrum. Methods Phys. Res. Sect. B Beam Interact. Mater. Atoms 375, 17-25 (2016)

21. C. Vivo-Vilches, J.M. López-Gutiérrez, R. Periáñez, C. Marcinko, F. Le Moigne, P. McGinnity, J.I. Peruchena, Villa-Alfageme M. Sci. Total Environ. 621, 376-386 (2018)

22. M. López-Lora et al., Sci. Total Environ. 708, 135222 (2020)

23. S. Padilla, J.M. López-Gutiérrez, D.M.R. Sampath, T. Boski, J.M. Nieto, M. García-León, J. Environ. Radioact. 189, 227-235 (2018)

24. H.-A. Synal et al., Nucl. Instrum. Methods Phys. Res. Sect. B Beam Interact. Mater. Atoms 259, 7-13 (2007)

25. L. Wacker et al., Radiocarbon 52(2-3), 252-262 (2010)

26. J.F. Gibaja et al., PLoS ONE 10(1), e0115505 (2015)

27. A. Rodríguez-Ramírez et al., Geomorphology 261, 103-118 (2016)

28. C. Ferro Vázquez et al., Radiocarbon 61(1), 101-130 (2019)

29. K.T. Uno et al., Proc. Natl. Acad. Sci. 110(29), 11736-11741 (2013)

30. J.F. Martín-Rodríguez et al., Sci. Rep. 5, 16298 (2015)

31. L. Fernandez-Maza et al., Appl. Radiat. Isot. 132, 79 (2018)

32. S. Rivera-Marrero et al., Curr. Radiopharm. 12(1), 58 (2019)

33. M. Balcerzyk et al., Nucl. Instrum. MethodsPhys. Res. Sect. A 873, 39 (2017)

34. E. Jimenez-Ortega et al., PLoS ONE 14(1), 18 (2019)

35. N.O. Nuñez et al., Nanomaterials 10(1), 149 (2020)

36. E.C.R. Leonel et al., Sci. Rep. 9, 1 (2019)

37. H. Martin, P. Martin-Holgado, Y. Morilla, L. Entrena, E. San-Millan, Radiat. Toler. Electr. 7, 163-174 (2018)

38. H. Martin, P. Martin-Holgado, P. Peris-Lopez, Y. Morilla, L. Entrena, Entropy 20(7), 513-524 (2018)

39. A. Lindoso, M. Garcia-Valderas, L. Entrena, Y. Morilla, P. Martin-Holgado, IEEE Trans. Nucl. Sci. 65, $1835-1842(2018)$

40. J. Praena-Rodriguez, P.F. Mastinu, M. Pignatari, J.M. Quesada-Molina, R. Capote-Noy et al., Nucl. Data Sheets 120, 205-207 (2014)

41. M. Romero-Expósito, S. Viñals, O. Ortega-Gelabert, B. Fernández, P. Jiménez-Bonilla, J. Praena, C. Domingo, Radiat. Prot. Dosim. 180, 80-84 (2018)

42. L. Irazola, F. Sanchez-Doblado, J. Praena-Rodriguez, B. Fernández, M. Macías et al., Appl. Radiat. Isot. 107, 330-334 (2016) 
43. D. Malagon, M.C. Jimenez-Ramos, F.J. Garcia-Lopez, S. Bota, G. Torrens et al., Microelectr. Reliab. 78, 38-45 (2017)

44. M.A. Millán-Callado, C. Guerrero, B. Fernández, M. Franconetti, J. Lerendegui-Marco, M. Macías, T. Rodríguez-González, J.M. Quesada, Springer Proc. Phys. 225, 153-154 (2019)

45. C. Lederer, F. Käppeler, M. Mosconi et al., Phys. Rev. C 85, 055809 (2012)

46. E. Chamizo, M. López-Lora, Nucl. Instrum. Methods Phys. Res. Sect. B 438, 198-206 (2019)

47. M. López-Lora, E. Chamizo, Nucl. Instrum. Methods Phys. Res. Sect. B 455, 39-51 (2019)

48. M. López-Lora et al., Sci. Total Environ. 142741 (2020) https://doi.org/10.1016/j.scitotenv.2020.142741

49. E. Chamizo et al., Sci. Total Environ. 740, 139993 (2020)

50. M.B.H. Breese, P.J.C. King, G.W. Grime, F. Watt, J. Appl. Phys. 72, 2097 (1992)

51. M.B.H. Breese, E. Vittone, G. Vizkelethy, P.J. Sellin, Nucl. Instr. Meth. B 264, 345-360 (2007)

52. E. Vittone et al., Nucl. Instr. Meth. B 372, 128-142 (2016)

53. J. Garcia Lopez, M.C. Jimenez-Ramos, M. Rodriguez-Ramos, J. Forneris, J. Ceballos. Nucl. Instr. Meth. B 371, 294-297 (2016)

54. J. Garcia Lopez, M.C. Jimenez-Ramos, M. Rodriguez-Ramos, J. Ceballos, F. Linez, J. Raisanen, Nucl. Instr. Meth. B 372, 143-150 (2016)

55. C. Cazzaniga et al., Nucl. Instr. Meth. B 405, 1-10 (2017)

56. C. Fleta et al., J. Instrum. 10, P10001 (2015)

57. M.C. Jiménez-Ramos et al., Radiat. Phys. Chem. 177, 10 (2020) 